\title{
Modeling of Scattering Cross Section for Mineral Aerosol with a Gaussian Beam
}

\author{
Wenbin Zheng $\mathbb{D i D}^{1}$ and Hong Tang $\mathbb{D}^{2,3}$ \\ ${ }^{1}$ College of Software Engineering, Chengdu University of Information Technology, Chengdu 610225, China \\ ${ }^{2}$ College of Engineering, Sichuan Normal University, Chengdu 610068, China \\ ${ }^{3}$ College of Metrology and Measurement Engineering, China Jiliang University, Hangzhou 310018, China
}

Correspondence should be addressed to Hong Tang; tangbenben@126.com

Received 31 December 2017; Accepted 28 January 2018; Published 28 March 2018

Academic Editor: Xiaoke Ku

Copyright ( 2018 Wenbin Zheng and Hong Tang. This is an open access article distributed under the Creative Commons Attribution License, which permits unrestricted use, distribution, and reproduction in any medium, provided the original work is properly cited.

Based on the generalized Lorenz Mie theory (GLMT), the scattering cross section of mineral aerosol within the Gaussian beam is investigated, and an appropriate modeling of the scattering cross sections for the real mineral aerosols including the feldspar, quartz, and red clay is proposed. In this modeling, the spheroid shape is applied to represent the real nonspherical mineral aerosol, and these nonspherical particles are randomly distributed within the Gaussian beam region. Meanwhile, the Monte Carlo statistical estimate method is used to determine the distributed positions of these random nonspherical particles. Moreover, a method for the nonspherical particles is proposed to represent the scattering cross section of the real mineral aerosols. In addition, the $T$ matrix method is also used to calculate the scattering cross sections of the spheroid particles in order to compare the scattering properties between the plane wave and the Gaussian wave. Simulation results indicate that fairly reasonable results of the scattering cross sections for the mineral aerosols can be obtained with this proposed method, and it can provide a reliable and efficient approach to reproduce the scattering cross sections of the real randomly distributed mineral aerosols illuminated by the Gaussian beam.

\section{Introduction}

The problem of light scattering by particles has been an important topic of research interest in the wide areas of applications [1-5]. Since the light scattering has been established, some researches have studied the electromagnetic light scattering of particles for the plane wave case [1]. And some common theories and methods have been utilized to analyze this problem. When the plane light wave is incident into the particle, the classical Mie theory, the discrete dipole approximation (DDA), the $T$ matrix method, and the finite difference time domain (FDTD) method can be used for sphere and nonsphere particles [6-9]. Nevertheless, none of those methods can be applied to analyze and calculate the scattering of particles for the nonplane waves such as the Gaussian beam incidence or a top-hat beam.

In recent years, with the development of laser technique and the expansion of its application areas, the laser has been used for the measuring of particle sizing and other particle prosperities. It is well known that the generalized Lorenz Mie theory (GLMT) proposed by
Gouesbet is a generalization of the Lorenz Mie theory for an arbitrary incident-shaped beam such as the Gaussian beam (laser in the fundamental mode TEM00) and the light sheet [10-12].

In this paper, the scattering cross sections for the practical nonspherical mineral aerosols are investigated, and modeling of the scattering cross sections for the feldspar, quartz, and red clay is conducted. Actually, the nonspherical calculations and measurements show significant differences from the sphere particles [13-16]. Here, we choose the spheroid to represent the nonspherical mineral aerosol in order to study the scattering cross sections of nonspherical particles within the Gaussian beam incidence.

\section{Theoretical Method and Calculations}

In the GLMT framework, the incident Gaussian beam field including the electric and the magnetic fields can be described by the Bromwich Scalar Potentials (BSP) in the spherical coordinate system $(r, \theta, \varphi)$ [10-12]. The field components are then found to be 


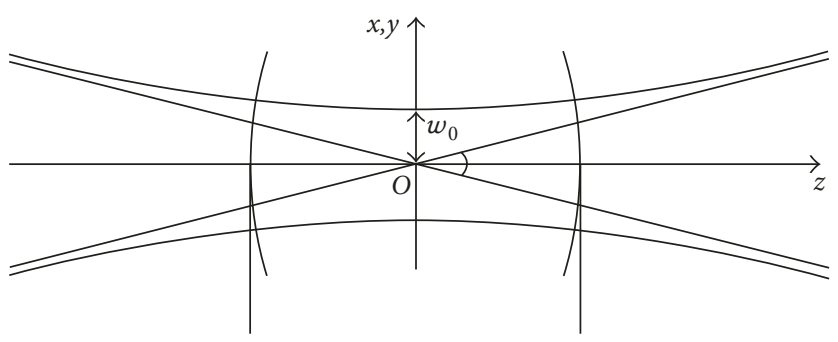

FIgURE 1: Geometry of coordinate of the incident Gaussian beam.

TABLE 1: Scattering cross section of sphere particle with the different position $(r=0.1, \lambda=0.5, m=1.33)$.

\begin{tabular}{lcccccc}
\hline Position & $(0,0,0)$ & $(0,1,0)$ & $(0,2,10.88)$ & $(0,0,10.88)$ & $(0.1,0.1,0.1)$ & $(0.1,0.1,1)$ \\
\hline$C_{\text {sca }}$ & $0.601405 E-14$ & $0.828364 E-15$ & $0.206664 E-15$ & $0.153682 E-14$ & $0.578179 E-14$ & $0.564966 E-14$ \\
\hline
\end{tabular}

TABLE 2: Scattering and absorbing cross section of sphere particle with the different location. $(r=0.1, \lambda=0.5, m=1.33+0.1 i)$.

\begin{tabular}{lcccccc}
\hline Position & $(0,0,0)$ & $(0,1,0)$ & $(0,2,10.88)$ & $(0,0,10.88)$ & $(0.1,0.1,0.1)$ & $(0.1,0.1,1)$ \\
\hline$C_{\text {sca }}$ & $0.579785 E-14$ & $0.798824 E-15$ & $0.199261 E-15$ & $0.148166 E-14$ & $0.557396 E-14$ & $0.544659 E-14$ \\
$C_{\text {abs }}$ & $0.105321 E-13$ & $0.147600 E-14$ & $0.365064 E-15$ & $0.270385 E-14$ & $0.101278 E-13$ & $0.989770 E-14$ \\
\hline
\end{tabular}

$(r=0.1, \lambda=0.5, m=1.33+0.1 i)$

$$
\begin{aligned}
E_{r}^{i} & =\frac{\partial^{2} U_{\mathrm{TM}}}{\partial r^{2}}+k^{2} U_{\mathrm{TM}}, \\
E_{\theta}^{i} & =\frac{1}{r} \frac{\partial^{2} U_{\mathrm{TM}}}{\partial r \partial \theta}-\frac{i \omega \mu}{r \sin \theta} \frac{\partial U_{\mathrm{TE}}}{\partial \varphi}, \\
E_{\varphi}^{i} & =\frac{1}{r \sin \theta} \frac{\partial^{2} U_{\mathrm{TM}}}{\partial r \partial \varphi}+\frac{i \omega \mu}{r} \frac{\partial U_{\mathrm{TE}}}{\partial \theta}, \\
H_{r}^{i} & =\frac{\partial^{2} U_{\mathrm{TE}}}{\partial r^{2}}+k^{2} U_{\mathrm{TE}}, \\
H_{\theta}^{i} & =\frac{i \omega \varepsilon}{r \sin \theta} \frac{\partial U_{\mathrm{TM}}}{\partial \varphi}+\frac{1}{r} \frac{\partial^{2} U_{\mathrm{TE}}}{\partial r \partial \theta}, \\
H_{\varphi}^{i} & =\frac{1}{r \sin \theta} \frac{\partial^{2} U_{\mathrm{TE}}}{\partial r \partial \varphi}-\frac{i \omega \varepsilon}{r} \frac{\partial U_{\mathrm{TM}}}{\partial \theta}
\end{aligned}
$$

where $U_{\mathrm{TM}}$ and $U_{\mathrm{TE}}$ are the transverse magnetic (TM) and transverse electric (TE) BSP, respectively; $i=\sqrt{-1} ; E^{i}$ and $H^{i}$ are called electric field and magnetic introduction field, respectively; $(r, \theta, \varphi)$ is the spherical coordinate system; $k$ is the wave number; $\omega$ is the angular frequency of the electromagnetic wave; and $\mu$ and $\varepsilon$ are the permeability and the permittivity of the medium, respectively.

The scattering cross section and extinction cross section of particle are evaluated by

$$
\begin{aligned}
C_{\mathrm{sca}}= & \frac{\lambda^{2}}{\pi} \sum_{n=1}^{\infty} \sum_{m=-n}^{n} \frac{2 n+1}{n(n+1)} \frac{(n+|m|) !}{(n-|m|) !} \\
& \times\left\{\left|a_{n}^{2}\right|\left|g_{n, \mathrm{TM}}^{m}\right|^{2}+\left|b_{n}^{2}\right|\left|g_{n, \mathrm{TE}}^{m}\right|^{2}\right\} \\
C_{\mathrm{ext}}= & \int_{0}^{\pi} \int_{0}^{2 \pi} \frac{1}{2} \operatorname{Re}\left(E_{\varphi}^{i} H_{\theta}^{s^{*}}+E_{\varphi}^{s} H_{\theta}^{i^{*}}-E_{\theta}^{i} H_{\varphi}^{s^{*}}-E_{\theta}^{s} H_{\varphi}^{i^{*}}\right) r^{2} \\
& \times \sin \theta d \theta d \varphi,
\end{aligned}
$$

where $g_{n \text {,TM }}^{m}$ and $g_{n \text {,TE }}^{m}$ are the generalized functions of GLMT, $a_{n}$ and $b_{n}$ are the scattering coefficient of Mie theory, and $E$ and $H$ are the magnetic and electric energy.

According to the GLMT, the particle is randomly located in the Gaussian beam, and the scattering properties are also determined by the location information in the beam. In Figure 1, the beam propagates alone the $z$ axis from negative $z$ to positive $z$, and the electric field component is essentially vibrating in the $x$ axis. The coordinate origin $o$ is the beam waist center, and its waist radius is $w_{0}$. The unit of the incident wavelength $\lambda$ and the particle radius $r$, as well as the beam waist radius $w_{0}$, is $\mu \mathrm{m}$ [17].

For a measured particle system, the particles can be distributed anywhere within the Gaussian beam [18]. Here, we define the particles in the semicircular region, that is, $0 \leq z \leq \sqrt{3} \pi w_{0}^{2} / \lambda, x^{2}+y^{2} \leq\left(2 w_{0}\right)^{2}$.

Table 1 shows the scattering cross section of the sphere particle with different location in the Gaussian beam. The particle radius $r$ is 0.1 , the incident wavelength $\lambda$ is 0.5 , the waist radius $w_{0}$ is 1 , and the relative complex reflective index of particle $m$ is 1.33 . The six positions in the Cartesian coordinate are $(0,0,0),(0,1,0),(0,2,10.88),(0,0,10.88),(0.1,0.1$, $0.1)$, and $(0.1,0.1,1)$. It is very obvious that the scattering cross section of sphere particle is also different when the position of particle is different. In Table 2, the scattering cross section and absorbing cross section of the sphere particle are calculated in the Gaussian beam. The relative complex reflective index of particle $m$ is $1.33+0.1 i$, the particle radius $r$ is 0.1 , the incident wavelength $\lambda$ is 0.5 , and the waist radius $w_{0}$ is 1 . We can see that the absorbing cross section of sphere particle is still different with the different position in the Gaussian beam.

In order to investigate the general average scattering cross sections of nonspherical mineral particles, three aerosols, that is, the feldspar, quartz, and red clay, are 


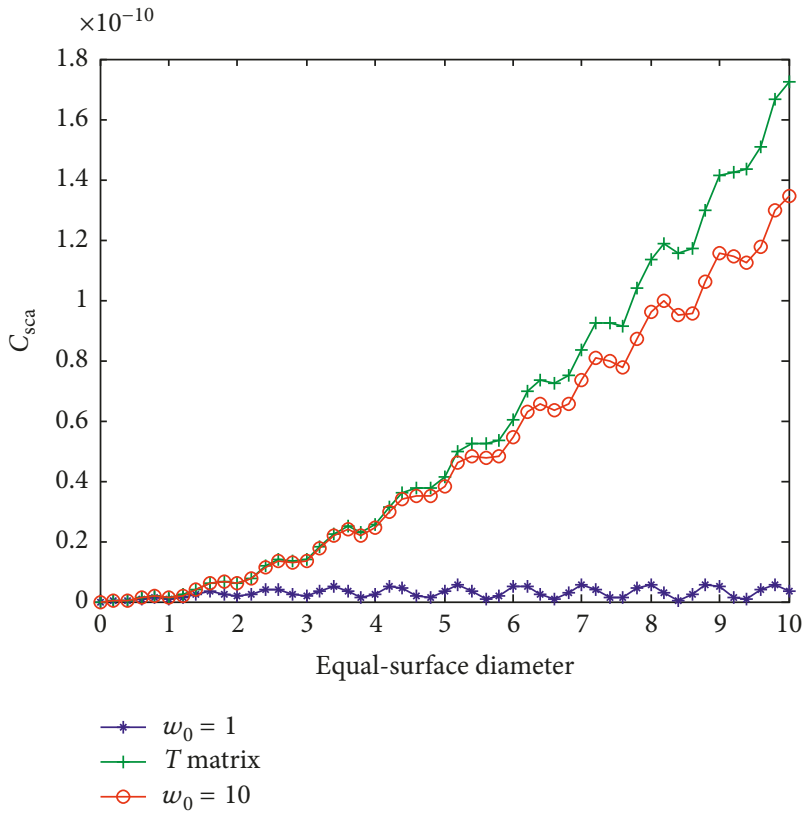

Figure 2: Scattering cross sections of quartz particles $(\lambda=0.5$, $m=1.54)$.

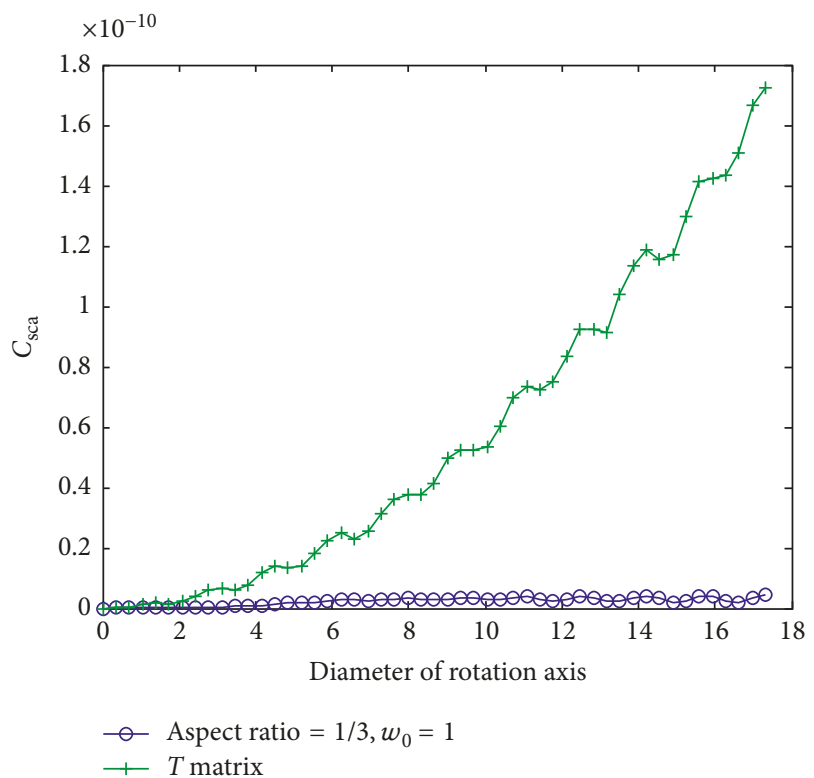

FIGURE 3: Scattering cross sections of quartz particles (aspect ratio $=1 / 3, \lambda=0.5, m=1.54$ ).

studied, and the spheroid model is used to represent the real nonspherical mineral particle. Since the scattering prosperities of particles within the Gaussian beam are related with the position information, the Monte Carlo statistical estimate method is used to determine the distributed positions of these random nonspherical particles $[19,20]$. After making the average of the position information, we can obtain the general location for the random particles, and then the average scattering cross section of particles is also calculated with the GLMT framework.

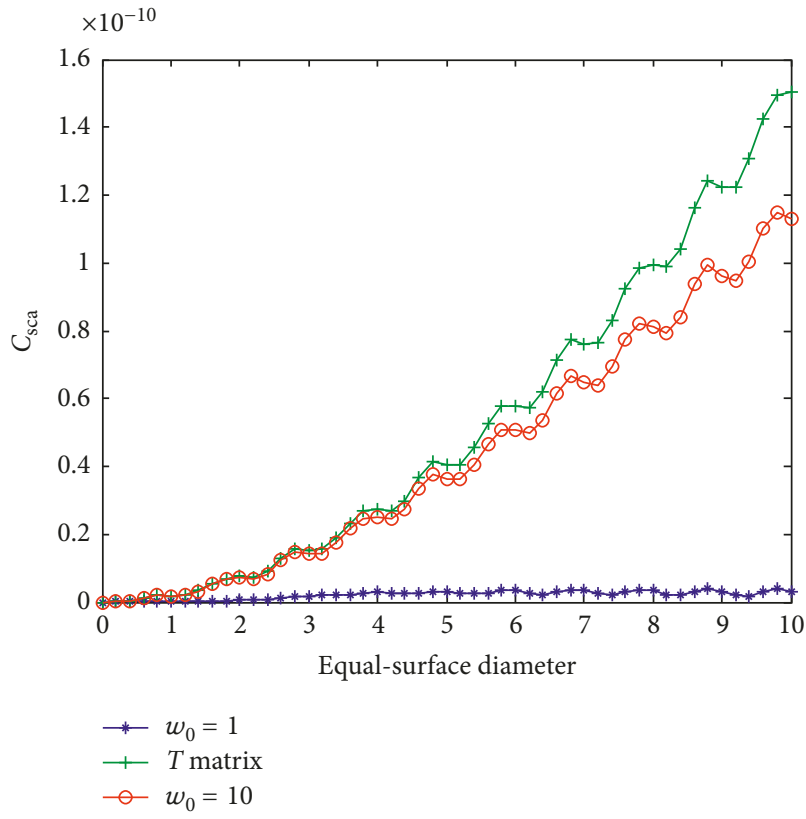

(a)

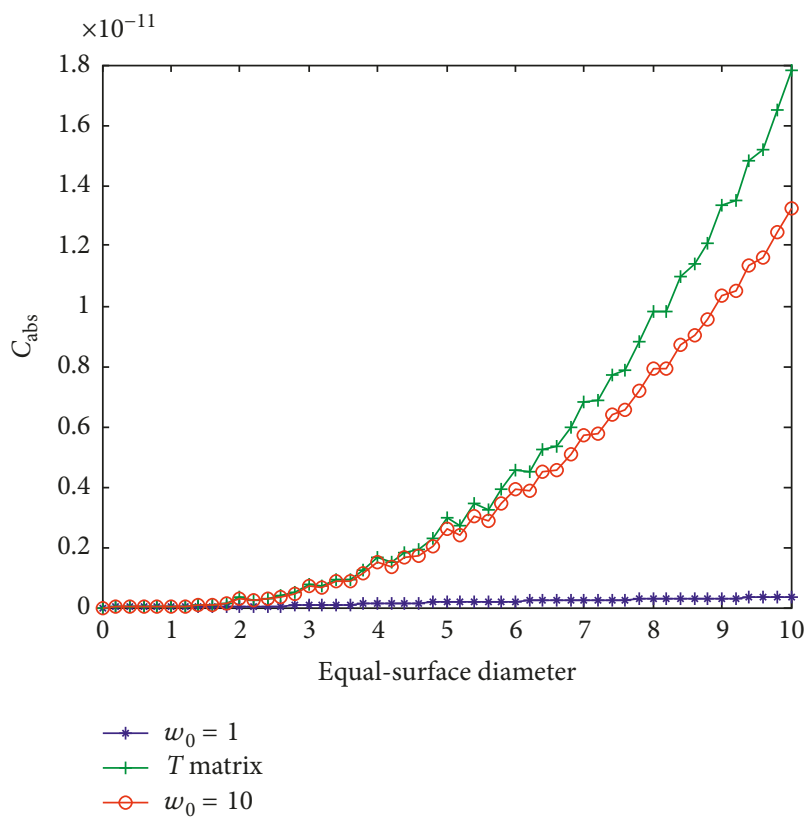

(b)

FIgURE 4: Scattering and absorbing cross sections of feldspar particles $(\lambda=0.5, m=1.5+0.001 i)$.

Figure 2 gives the scattering cross sections of spheroid quartz particles with different sizes. For a spheroid, there are two parameters representing its shape, that is, the aspect ratio and the radius of rotation axis. Here, we use the equal-surface area sphere to represent the spheroid particle, and the equalsurface diameter can be calculated by the spheroid particle. In Figure $2, w_{0}$ is equal to 1 and 10 , respectively, and the Tmatrix method is also used to obtain the scattering cross sections of spheroid particles for the plane wave as a comparison [21]. The relative complex reflective index of quartz particle $m$ is 


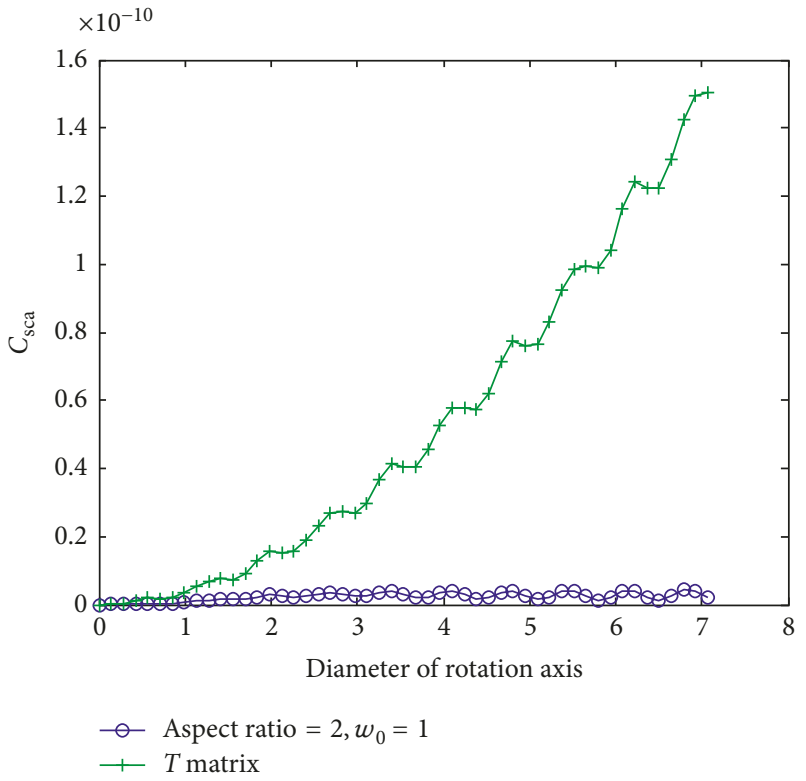

(a)

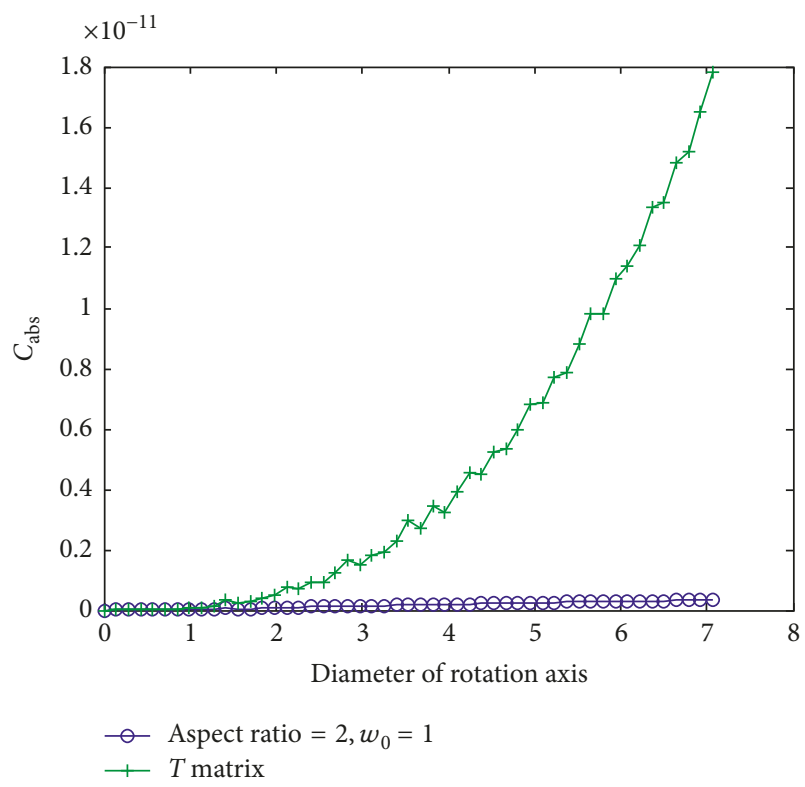

(b)

Figure 5: Scattering and absorbing cross sections of feldspar particles (aspect ratio $=2, \lambda=0.5, m=1.5+0.001 i$ ).

1.54 , and the incident wavelength $\lambda$ is 0.5 . We can see that serious differences are occurred between the Gaussian beam and the plane wave, and the differences are decreasing with larger $w_{0}$. That is because the Gaussian beam gradually becomes the plane wave when $w_{0}$ is infinite. Figure 3 describes the scattering cross sections of spheroid quartz particles with aspect ratio $=1 / 3$ and $w_{0}=1$. The parameter in the horizontal axis is the diameter of rotation axis, and the $T$ matrix method is also used to obtain the scattering cross sections of spheroid particles for the plane wave as a comparison.

Figure 4 gives the scattering and absorbing cross sections of feldspar particles. Figure 5 is the scattering and

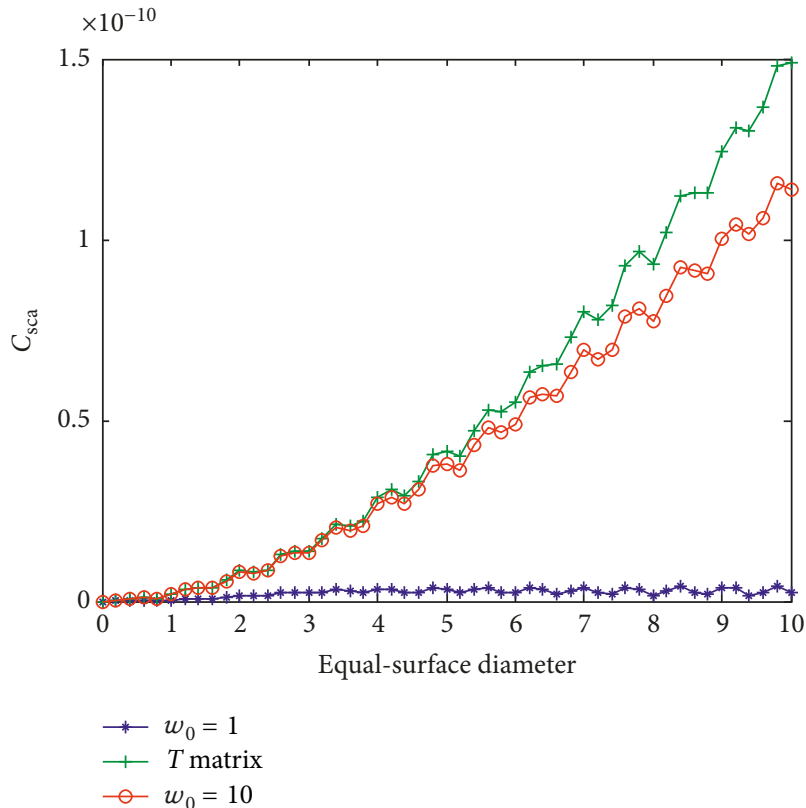

(a)

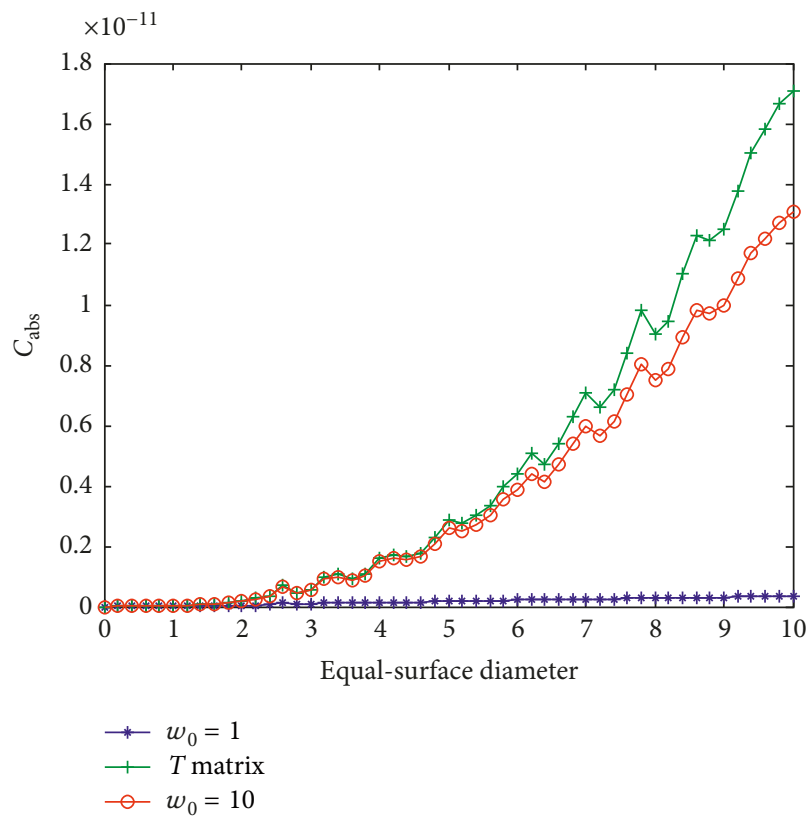

(b)

FIGURE 6: Scattering and absorbing cross sections of red clay particles $(\lambda=0.5, m=1.7+0.001 i)$.

absorbing cross sections of feldspar particles with aspect ratio $=2$. For the feldspar particles, the imaginary part of the complex reflective index is not zero, and then the absorbing cross sections of feldspar particles can be calculated. With the increasing equal-surface diameter or the diameter of rotation axis, the differences between the Gaussian beam and plane wave are enlarged.

Figure 6 gives the scattering and absorbing cross sections of red clay particles. Figure 7 is the scattering and absorbing cross sections of red clay particles with aspect ratio $=1 / 2$. For 


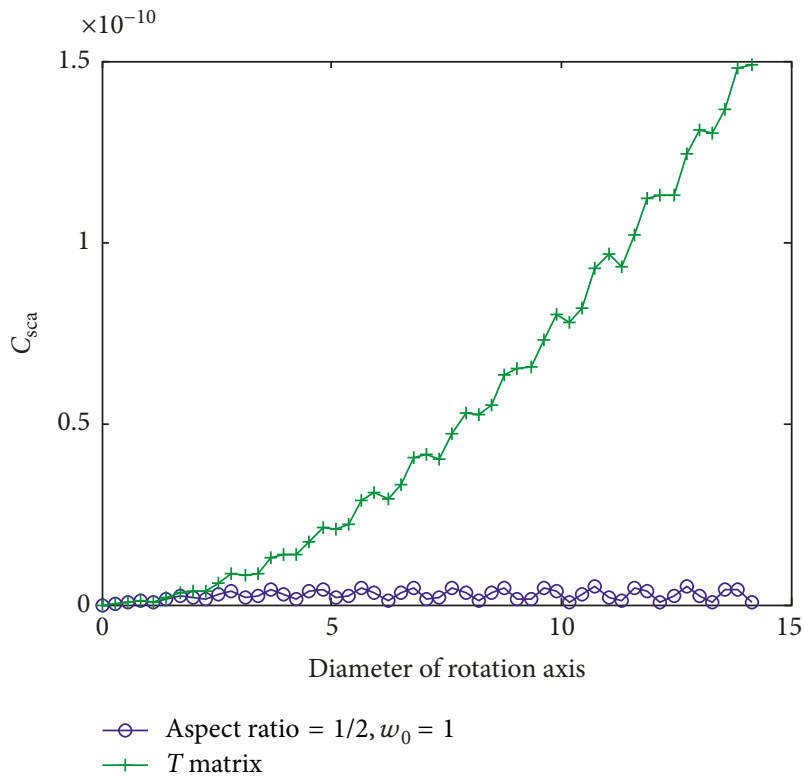

(a)

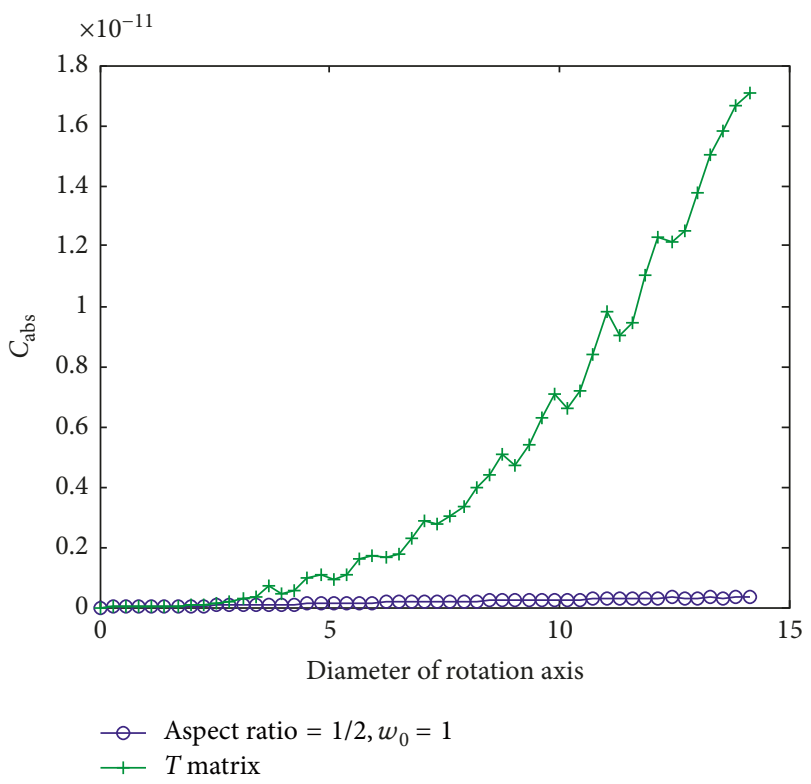

(b)

Figure 7: Scattering and absorbing cross sections of red clay particles (aspect ratio $=1 / 2, \lambda=0.5, m=1.7+0.001 i$ ).

the red clay particles, the real part of the complex reflective index is larger than that of the feldspar particles and the quartz particles. The differences are relatively small between the Gaussian beam and plane wave with $w_{0}=10$, compared with the differences of scattering and absorbing cross sections of the feldspar particles and the quartz particles.

Figures 8 and 9 show the scattering and absorbing cross sections of spheroid particles with $1.5+0.01 i$. In Figure 9, the incident wavelength is 1.2. According to this simulation, there are still differences between the Gaussian beam and plane wave, and the differences are smaller with larger incident wavelength.

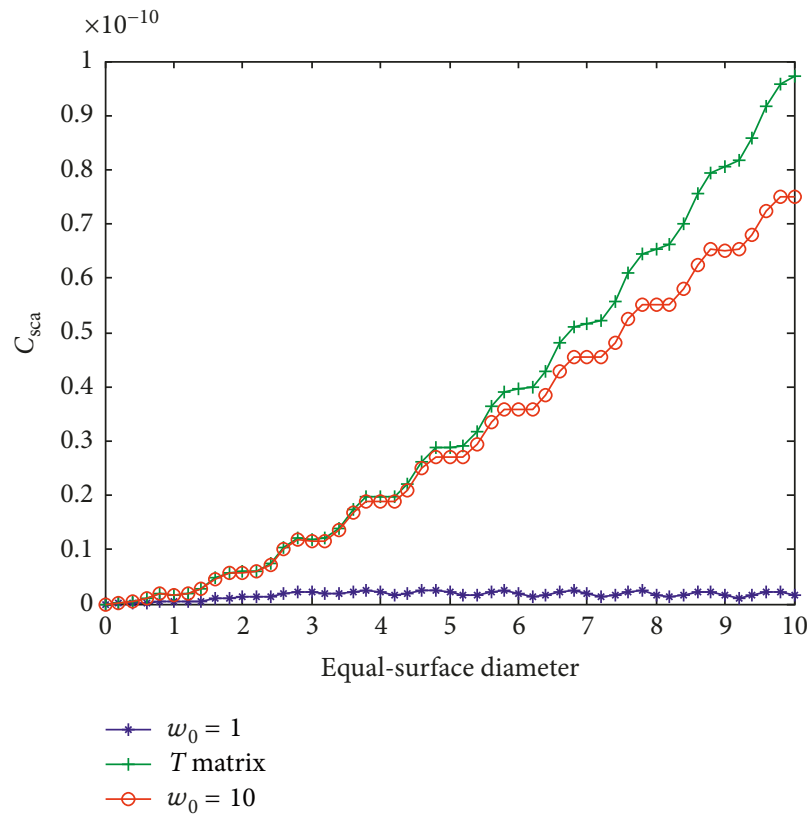

(a)

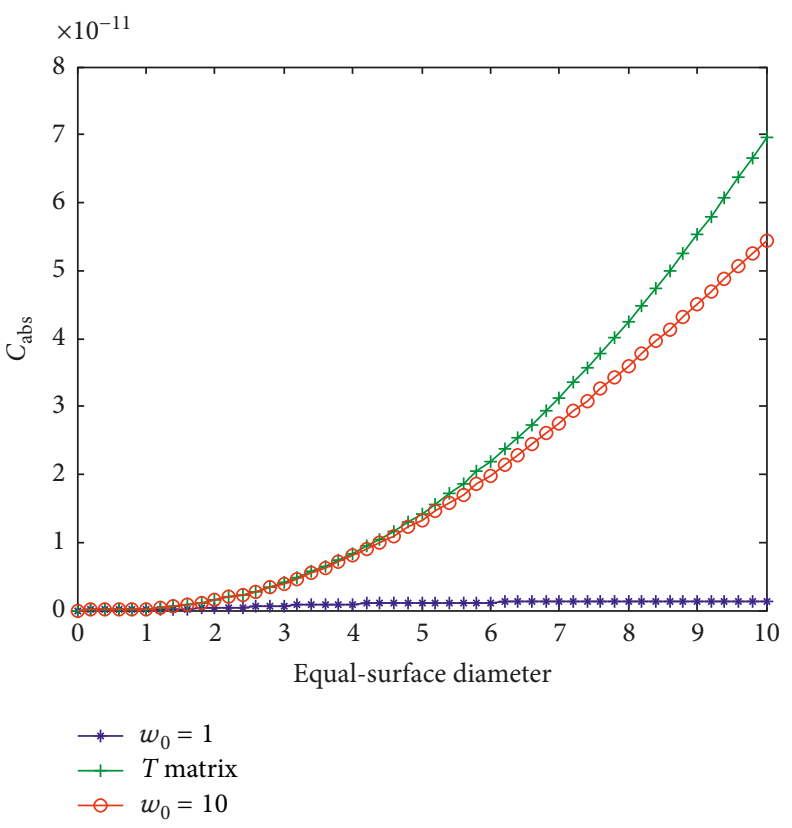

(b)

FiguRE 8: Scattering and absorbing cross sections of spheroid particles $(\lambda=0.5, m=1.5+0.01 i)$.

\section{Conclusions}

In this paper, the scattering cross sections of nonspherical mineral particles are investigated with in the Gaussian beam based on the GLMT. In the framework of GLMT, the general location information is statistic by the Monte Carlo statistical estimate method, and the scattering cross sections of spheroid particles including the feldspar, quartz, and red clay are calculated. Actually, the spheroid shape can represent the nonspherical feldspar, quartz, and red clay 


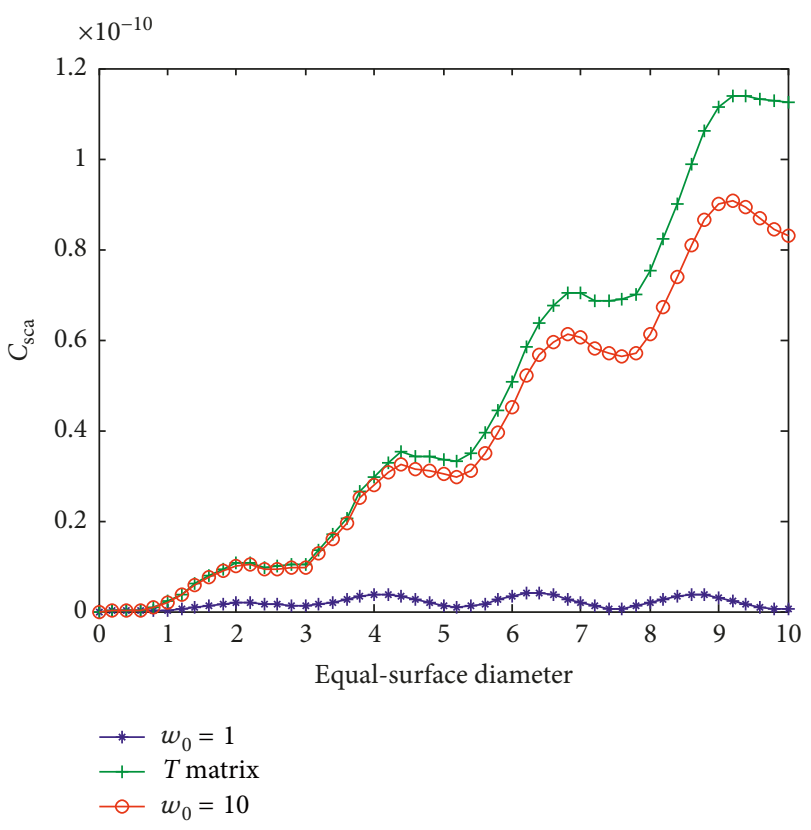

(a)

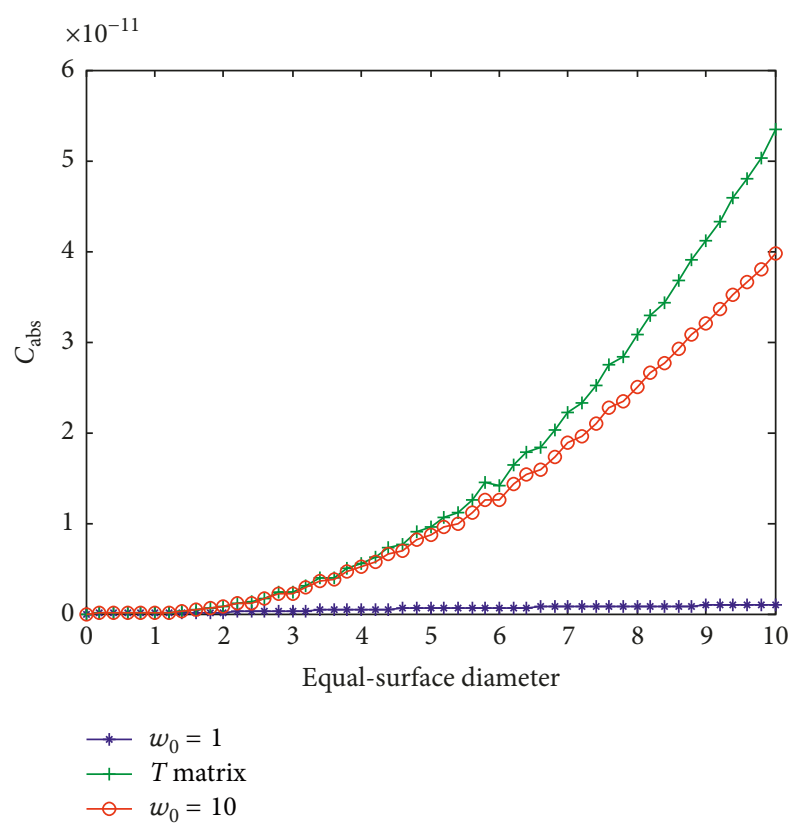

(b)

FIGURE 9: Scattering and absorbing cross sections of spheroid particles $(\lambda=1.2, m=1.5+0.01 i)$.

particles with good accuracy. In order to research the scattering of spheroid particle with more efficiency, a sphere of the same surface area as the spheroid is used to calculate the scattering cross sections of spheroid particles within the Gaussian beam, and then the scattering cross sections of spheroid particles are also calculated within the plane wave incidence. The results show that the scattering cross sections and absorbing cross sections of spheroid are different from the plane wave, and the differences are more obvious with the increasing diameters. Meanwhile, the incident wavelength and the complex reflective index of mineral particles also have effects on the scattering cross sections of nonspherical mineral particles.

\section{Conflicts of Interest}

The authors declare that they have no conflicts of interest.

\section{Acknowledgments}

This research was supported by the Zhejiang Province Natural Science Funds (LY15A020003), the Major Project of Education Department in Sichuan (18ZA0409), the Applied Basic Research Project of Sichuan Province, and the Open project Fund of Laboratory of Meteorological Information Sharing and Data Mining (QGX18009). The authors are grateful to Gérard Gouesbet from INSA de Rouen, France, for useful discussions on GLMT and some help.

\section{References}

[1] J. A. Lock and G. Gouesbet, "Rigorous justification of the localized approximation to the beam-shape coefficients in generalized Lorenz-Mie theory. I. On-axis beams," Journal of the Optical Society of America A, vol. 11, no. 9, pp. 2503-2513, 1994.

[2] K. N. Liou, Y. Takano, and P. Yang, "Light absorption and scattering by aggregates: application to black carbon and snow grains," Journal of Quantitative Spectroscopy and Radiative Transfer, vol. 112, no. 10, pp. 1581-1595, 2011.

[3] Z. M. Li, J. Shen, X. M. Sun, and Y. J. Wang, "Nanoparticle size measurement from dynamic light scattering data based on an autoregressive model," Laser Physics Letters, vol. 10, no. 9, p. 095701, 2013.

[4] M. Z. Yu, J. Z. Lin, and T. L. Chan, "A new moment method for solving the coagulation equation for particles in Brownian motion," Aerosol Science \& Technology, vol. 42, no. 9, pp. 705-713, 2008.

[5] M. Yu and J.Z. Lin, "Taylor expansion moment method for agglomerate coagulation due to Brownian motion in the entire size regime," Journal of Aerosol Science, vol. 40, no. 6, pp. 549-562, 2009.

[6] J. Tientong, S. Garcia, C. R. Thurber, and T. D. Golden, "Synthesis of nickel and nickel hydroxide nanopowders by simplified chemical reduction," Journal of Nanotechnology, vol. 2014, Article ID 193162, 6 pages, 2014.

[7] Z. F. Li, H. Li, H. Zhang, X. N. Lin, and W. R. Chen, "Extracting optical scattering properties on the basis of phase contrast images for diagnosing stomach cancer," Laser Physics, vol. 23, no. 4, p. 045605, 2013.

[8] Y. J. Hu, R. Sun, Z. X. Huang, and X. L. Wu, "The repulsive Casimir force with metallic ellipsoid structure," Journal of Nanotechnology, vol. 2016, Article ID 1746908, 5 pages, 2016.

[9] W. B. Sun, Y. X. Hu, C. Weimer, K. Ayers, R. R. Baize, and T. Lee, "A FDTD solution of scattering of laser beam with orbital angular momentum by dielectric particles: far-field characteristics," Journal of Quantitative Spectroscopy and Radiative Transfer, vol. 188, pp. 200-213, 2017.

[10] G. Gouesbet, "T-matrix formulation and generalized Lorenz-Mie theories in spherical coordinates," Optics Communications, vol. 283, no. 4, pp. 517-522, 2010. 
[11] Y. P. Han, H. Y. Zhang, and G. X. Han, "The expansion coefficients of arbitrary shaped beam in oblique illumination," Optics Express, vol. 15, no. 2, pp. 735-747, 2007.

[12] K. F. Ren, G. Grehan, and G. Gouesbet, "Evaluation of laser sheet beam shape coefficients in generalized Lorenz-Mie theory by using a localized approximation," Journal of the Optical Society of America A, vol. 11, no. 7, pp. 2072-2080, 1994.

[13] M. Kahnert, T. Nousiainen, and B. Veihelmann, "Spherical and spheroidal model particles as an error source in aerosol climate forcing and radiance computations: a case study for feldspar aerosols," Journal of Geophysical Research: Atmospheres, vol. 110, no. 18, pp. 1-12, 2005.

[14] J. Z. Lin, X. Shi, and Z. J. You, "Effects of the aspect ratio on the sedimentation of a fiber in Newtonian fluids," Journal of Aerosol Science, vol. 34, no. 7, pp. 909-921, 2003.

[15] J. Z. Lin, X. Shi, and Z. S. Yu, "The motion of fibers in an evolving mixing layer," International Journal of Multiphase Flow, vol. 29, no. 8, pp. 1355-1372, 2003.

[16] J. Z. Lin, W. F. Zhang, and Z. S. Yu, "Numerical research on the orientation distribution of fibers immersed in laminar and turbulent pipe flows," Journal of Aerosol Science, vol. 35, no. 1, pp. 63-82, 2004.

[17] D. P. Chrissoulidis and E. Richalot, "Wave-amplitude synthesis applied to Gaussian-beam scattering by an off-axis sphere," Journal of the Optical Society of America, vol. 34, no. 4, pp. 558-567, 2017.

[18] H. Tang and J. Z. Lin, "Scattering of randomly distributed spherical particles illuminated with a Gaussian beam," Laser Physics Letters, vol. 12, no. 4, p. 046001, 2015.

[19] P. Lai, Z. L. Cai, J. P. Pignol et al., "Monte Carlo simulation of radiation transport and dose deposition from locally released gold nanoparticles labeled with ${ }^{111} \mathrm{In},{ }^{177} \mathrm{Lu}$ or ${ }^{90} \mathrm{Y}$ incorporated into tissue implantable depots," Physics in Medicine and Biology, vol. 62, no. 22, pp. 8581-8599, 2017.

[20] R. M. Saeb, Y. Mohammadi, H. Rastin, T. S. Kermaniyan, and A. Penlidis, "Visualization of bivariate sequence length-chain length distribution in free radical copolymerization," Macromolecular Theory and Simulations, vol. 26, no. 5, p. 1700041, 2017.

[21] M. I. Mishchenko and L. D. Travis, "Capabilities and limitations of a current FORTRAN implementation of the T-matrix method for randomly oriented, rotationally symmetric scatterers," Journal of Quantitative Spectroscopy and Radiative Transfer, vol. 60, no. 3, pp. 309-324, 1998. 


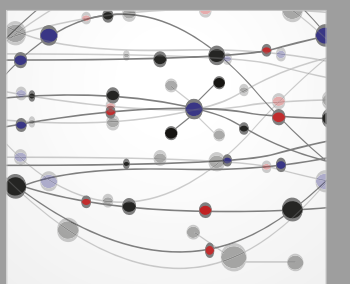

The Scientific World Journal
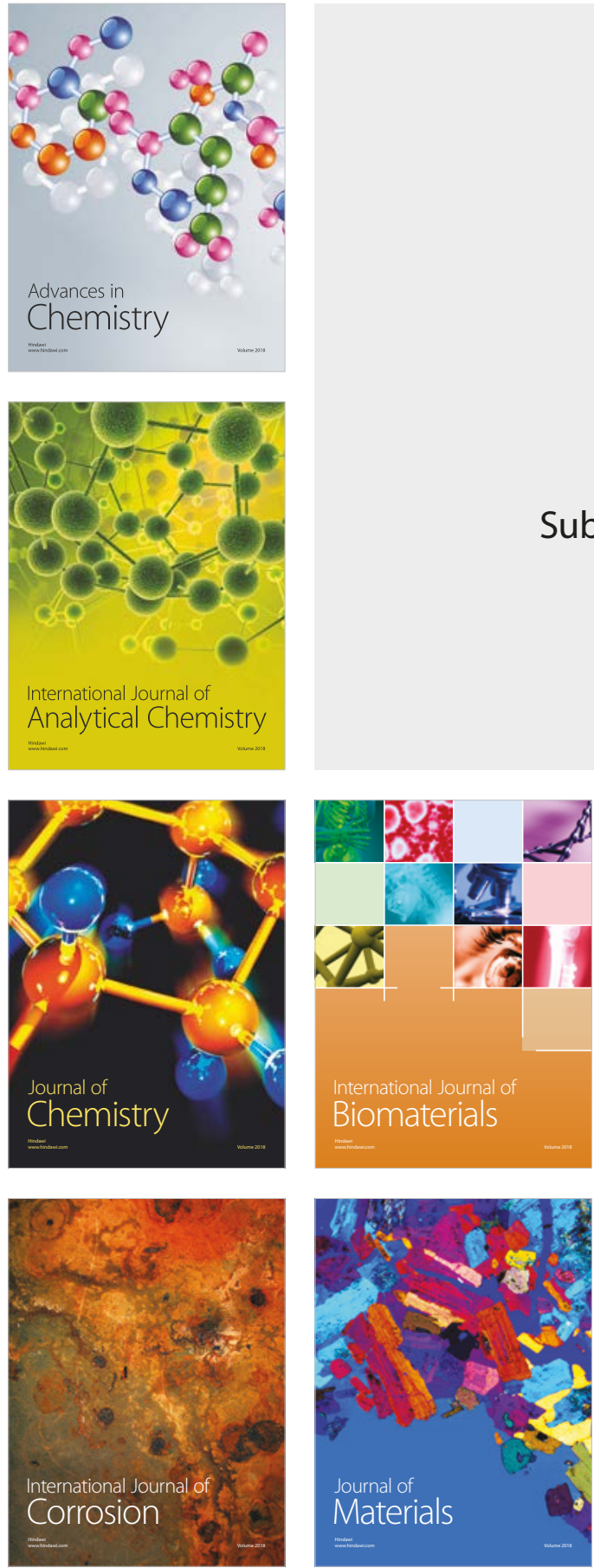

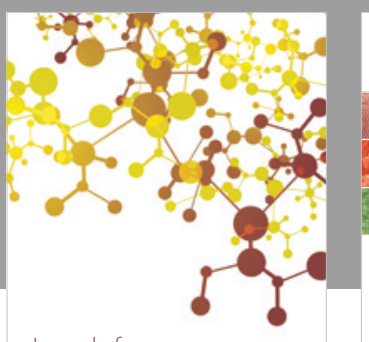

Journal of

Applied Chemistry
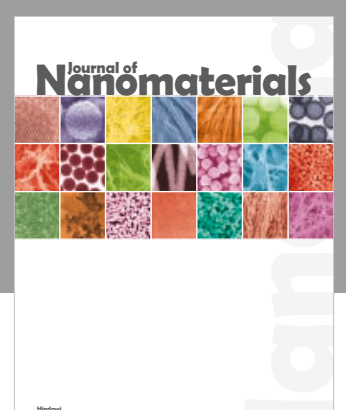

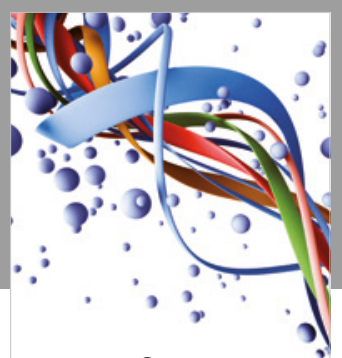

Scientifica

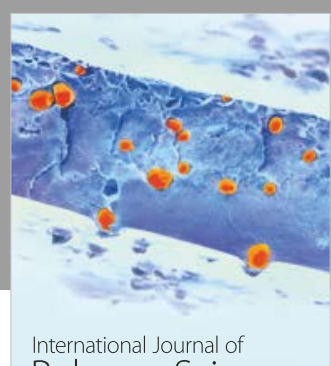

Polymer Science

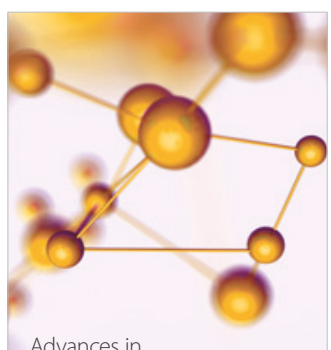

Physical Chemistry
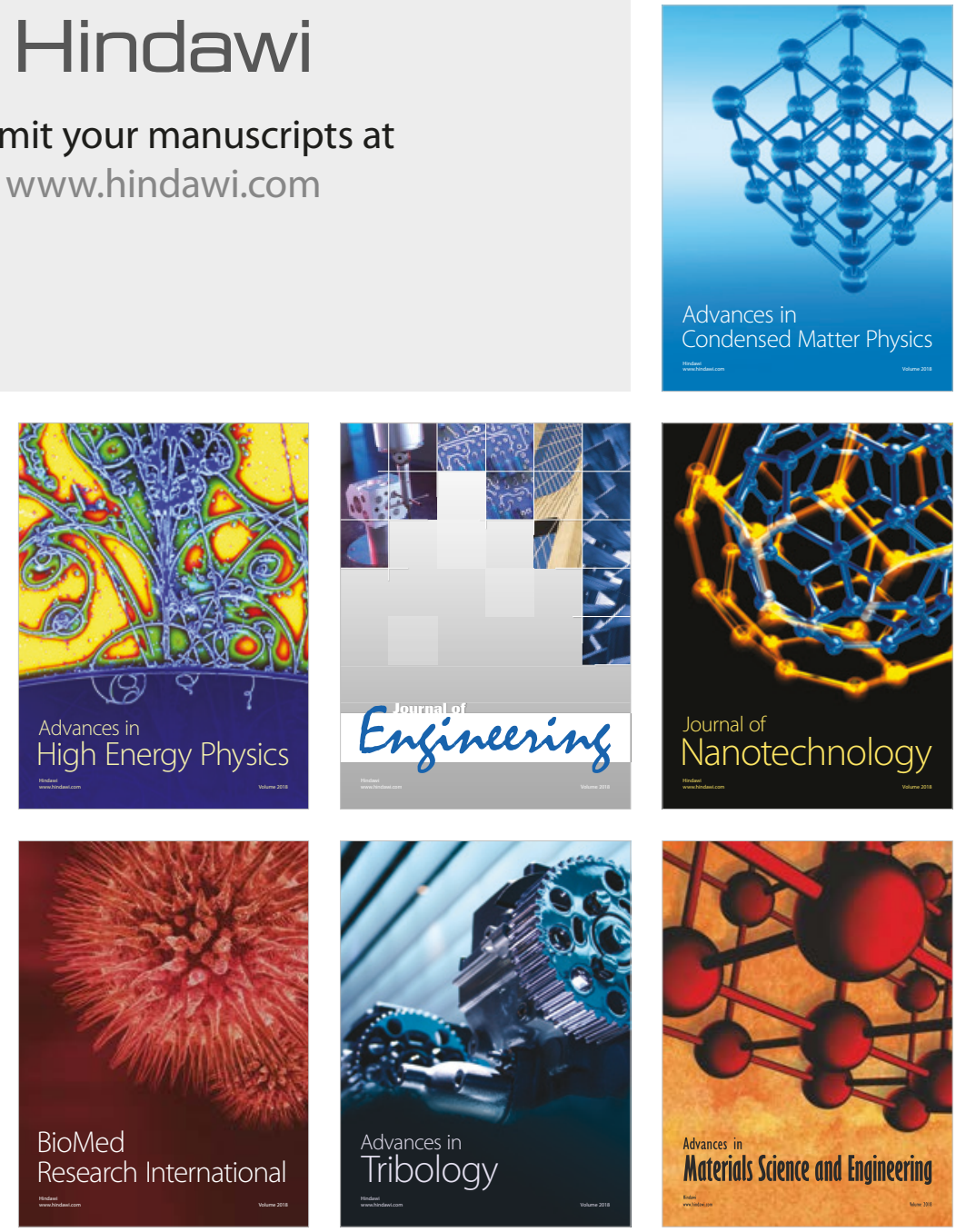